\title{
$N 91-16902$
}

\section{RADIO LOUD FAR-INFRARED GALAXIES}

\author{
Arjun Dey \\ Astronomy Department, University of California at Berkeley \\ Wil van Breugel \\ Institute of Geophysics and Planetary Physics, LLNL, Livermore \\ Joseph C. Shields \\ Astronomy Department, University of California at Berkeley
}

\begin{abstract}
We present the first results of a multiwavelength study of IRAS galaxies with excess radio emission. The sample was selected by cross-correlating the IRAS Faint Source Survey (for $|\mathrm{b}| \geq 50^{\circ}$ ) and the Point Source Catalogue (for $10^{\circ}<|\mathrm{b}|<50^{\circ}$ ) with the Texas radio survey. Recent optical (imaging and spectroscopic) and radio (VLA) observations are discussed. These observations will be used to investigate possible connections between radio galaxy activity, star formation and galaxy interactions.
\end{abstract}

\section{Introduction}

Increasing evidence exists for a possible evolutionary connection between galaxy interactions, central starbursts and activity of galaxy nuclei (e.g. Heckman 1987; Norman and Scoville 1988). A popular scenario is that tidal interactions between galaxies trigger a central starburst in one of them. This starburst then evolves into a black hole and/or increases its fuelling rate, resulting in enhanced activity of the galaxy nucleus. To a large extent such models have been motivated by studies of galaxies discovered by the IRAS survey.

Optical imaging of ultraluminous far-infrared (FIR) galaxies has shown that many are interacting with nearby companions. Interacting systems are also frequently found associated with Seyferts, powerful radio galaxies and quasars. These galaxies are often FIR emitters and can be selected from IRAS catalogs by their "warm" colors. Large populations of relatively young stars, inferred from stellar absorption lines, exist in many ultraluminous FIR galaxies (e.g. Armus et al. 1990). Similar evidence for the other classes of galaxies is more difficult to obtain, but some good examples are known such as the radio galaxy 3C 459 (Miller 1981), and the quasar $3 \mathrm{C} 48$ (Boroson and Oke 1982; Neugebauer et al. 1982). The interpretation of emission-line data is more ambiguous and different sources of ionization (H II regions, LINERs and AGNs) might contribute (Armus et al. 1990). Bulk outflow, either in the form of hot winds or collimated jets, occurs in all above mentioned classes of objects. Taken together one may think that there is some observational support for a "grand unified theory of active galaxies", but better evidence is needed. In order to study various FIR/radio/optical relationships we have defined a sample of sources from the IRAS Point Source Catalog (PSC) and Faint Source Survey (FSS). We have not used any additional IR color, luminosity or optical selection criteria.

\section{Sample Selection and Observations}

Our radio sources were taken from the Texas catalogue at $365 \mathrm{MHz}$ (Douglas et al. 1980). This catalogue is $\sim 90 \%$ complete to a flux density limit of $250 \mathrm{mJy}$ for sources of angular sizes $\lesssim 1$ arcminute, and the version that we used covered the region $-35^{\circ}<\delta<40^{\circ},|\mathrm{b}|>10^{\circ}$. Sources from this catalogue were compared with the IRAS FSS (for $|b|>50^{\circ}$; an overlap of $\sim 1.9$ steradians) and the IRAS PSC (for $|b|<50^{\circ}$; an overlap of $\sim 4.7$ steradians) for $60 \mu$ sources with positional 


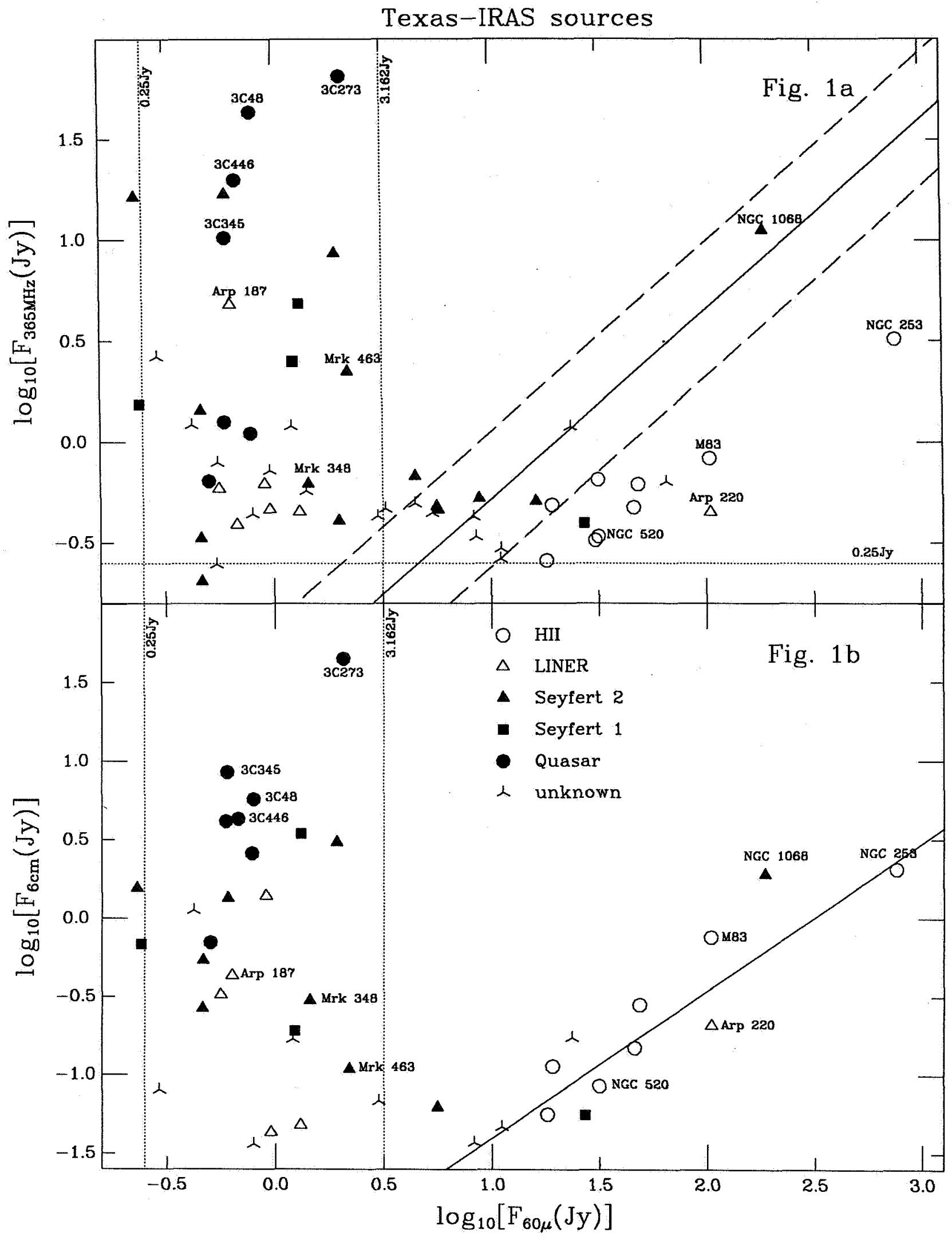


coincidence within $60^{\prime \prime}$. This resulted in 67 sources from the PSC and 32 sources from the FSS. The Texas radio source positions are accurate to about $\mathbf{1}^{\prime \prime}$, allowing optical identifications to be found on Palomar Sky Survey prints. A total of 60 IRAS/radio/optical identifications were found, of which 34 had $\log \left[\mathrm{F}_{60 \mu}(\mathrm{Jy})\right]<0.5$. The remaining 26 objects are mostly well studied nearby bright galaxies.

All sources with $\log \left[\mathrm{F}_{60 \mu}(\mathrm{Jy})\right]<0.5$ were mapped at $21 \mathrm{~cm}$ using the VLA in the A-array configuration. We obtained optical images of these sources at Lick Observatory using the 1-metre Nickel and 3-metre Shane telescopes. Optical spectra were also obtained at a resolution of $\sim 15 \AA$.

\section{Preliminary Results}

Figure $1 a$ shows the $365 \mathrm{MHz}$ flux density (from the Texas catalogue) plotted against the $60 \mu$ flux density (from IRAS) for the sources in our sample. Figure $1 b$ shows the same plot, but using the $6 \mathrm{~cm}$ flux densities for the sources as listed in the catalogue constructed by Becker et al. 1990 from the Condon et al. (1989) $6 \mathrm{~cm}$ Green Bank survey, and the Parkes catalogue. The diagonal lines in Figure $1 a$ indicate where the $6 \mathrm{~cm}-60 \mu$ correlation for spiral galaxies from de Jong et al. (1985) would fall when shifted to $365 \mathrm{MHz}$ using a spectral index of 0.7 (lower dashed line), 1.0 or $1.3\left(\mathrm{~S}_{\nu} \propto \nu^{-\alpha}\right)$. All the objects with $\log _{10}\left[\mathrm{~S}_{60 \mu}(\mathrm{Jy})\right]<0.5$ lie above this correlation; we refer to these as "radio loud".

We also note that the actual $365 \mathrm{MHz}$ flux densities for $\log _{10}\left[\mathrm{~S}_{60 \mu}(\mathrm{Jy})\right]>0.5$ galaxies are considerably below their predicted values using a typical (high frequency) spectral index of 0.7 or larger. This effect is clearly demonstrated by comparing Figure $1 a$ with Figure 1b. The diagonal line in Figure $1 b$ shows the de Jong et al. $6 \mathrm{~cm}-60 \mu$ correlation which, as expected, agrees with the IR-bright galaxies sources in our sample. Many of these sources are well studied galaxies (Arp 220, NGC 253, NGC 1052, NGC 1068, etc.). In the case of Arp 220 a possible explanation for the flattening of the radio spectrum at lower frequencies is free-free absorption by dense gas (Sopp and Alexander 1989). This may also be the case for the other IR-bright galaxies. Further radio spectral information of the sources in our sample will be needed to investigate the generality of this phenomenon.

Figure 2 shows an IRAS color-color plot for the sources in our sample. The dashed lines outline the regions enclosing the mean ( \pm one standard deviation) spectral indices for normal spirals, starbursts, and Seyfert 2 galaxies (Sekiguchi 1987). Vertical and horizontal arrows indicate that only upper limits to the $25 \mu$ and $100 \mu$ flux densities were available. Although our sample was not selected on the basis of IR colors, most of our sources exhibit IR colors typical of active galaxies.

We have also examined the radio - IR $(60 \mu)$ luminosity distribution of our sample (not shown). Our sample has a high median IR luminosity $\left(\mathrm{L}_{60 \mu} \sim 4 \times 10^{11} \mathrm{~L}_{\odot}, \mathrm{H}_{\circ}=50 \mathrm{~km} / \mathrm{s} / \mathrm{Mpc}\right)$, quite different from the $\mathrm{L}_{60 \mu}$ distribution of the Bright Galaxy Sample (Soifer et al. 1989), but more similar to that of Seyferts and quasars (Neugebauer et al. 1986). Sanders et al. (1988) have suggested that IR-ultraluminous galaxies like Arp 220 might be powered by quasar-like nuclei. We find very little overlap between our radio-loud sample and that of Sanders et al. Only Arp 220 is a strong enough radio emitter to be common to both samples over the appropriate area of sky. A more detailed analysis of the IR colors and optical spectra of our radio-loud FIR galaxies is needed to see whether there are other differing properties.

Of the radio loud FIR galaxies, $40 \%$ have flat radio spectra $(\alpha \lesssim 0.5)$ and very compact radio cores. Of the remaining $60 \%$ with relatively steep spectra, $80 \%$ have their dominant radio emission on small angular scales $\left(\lesssim 5^{\prime \prime}\right)$. These sources are entirely embedded within their parent galaxies and are ideal sources in which to study the interaction between radio sources and their surrounding interstellar medium. One example, Arp 187, is shown in Figure 3 which includes an R-band image (3a), $21 \mathrm{~cm}$ VLA map (3b) and optical spectrum (3c). Note that $\mathrm{H} \beta$ appears in absorption, indicating the possible presence of a large young stellar population in the galaxy. 


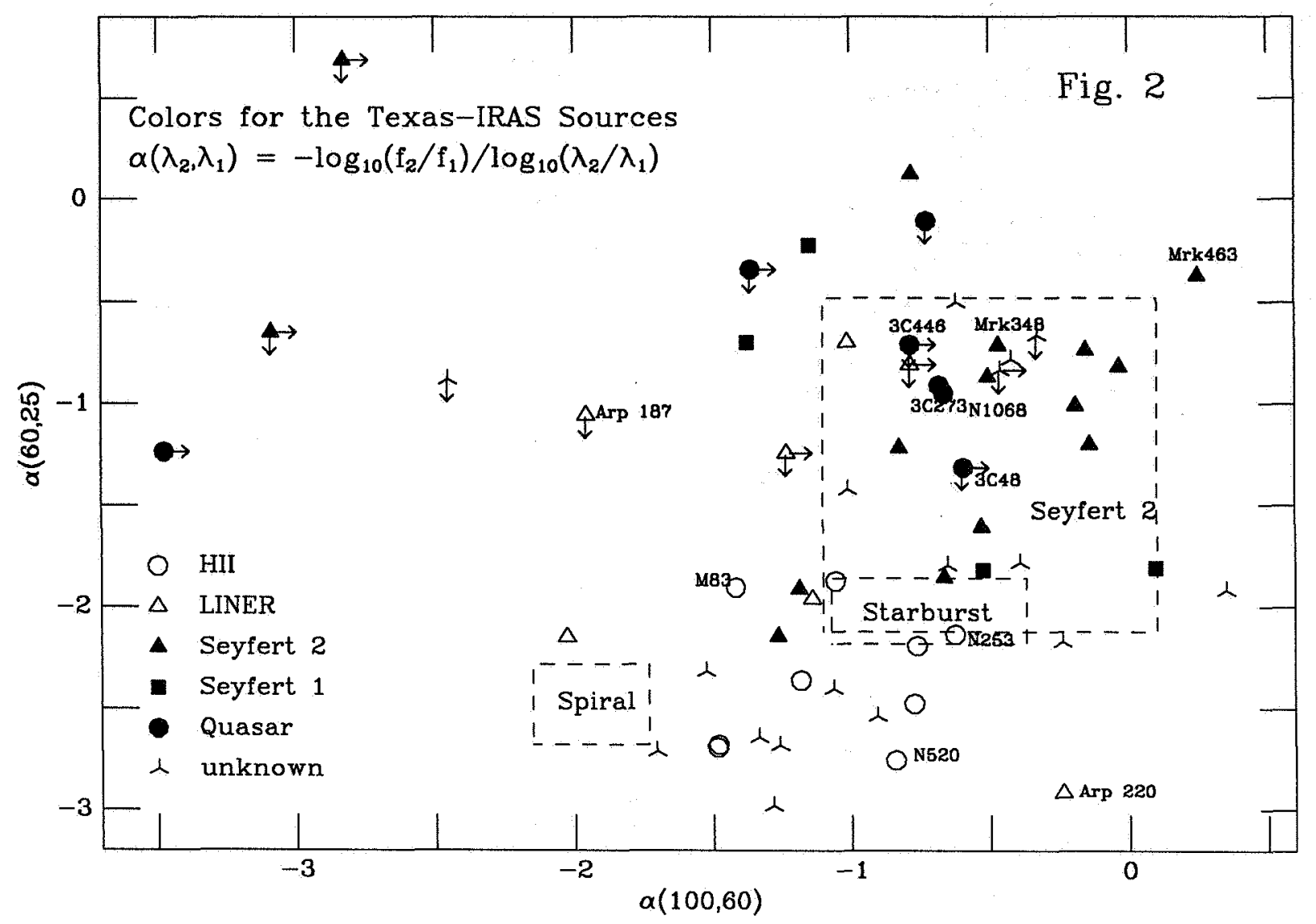

Of the galaxies in our radio-loud sample, 7 are LINERs, 3 are Seyfert 1 galaxies, 10 are Seyfert 2 or radio galaxies, and 7 are quasars. Thus our sample encompasses a large variety of galaxy activity. More detailed studies, and increasing the sample size, may help us better understand possible links between these classes of objects and starburst activity.

\section{References}

Armus, L. Heckman, T. M. and Miley, G. K., 1990, preprint

Becker, R. H., White, R. L. and Edwards, A. L., 1990, preprint

Boroson, T. A., and Oke, J. B., 1982, Nature, $296,397$.

Condon, J. J., Broderick, J. J. and Seielstad, G. A., 1989, Astron. J., 97,1064.

de Jong, T., Klein, U., Wielebinski, R., and Wunderlich, E., 1985,Astron. Astrophys., 147,L6

Douglas, J. N., Bash, F. N., Torrence, G. W., and Wolfe, C., 1980,Univ. Texas Publ. Astron. no.17

Heckman, T. M. , 1987, in Starbursts and Galaxy Evolution, T. X. Thuan, T. Montmerle, J. T. T. Van, Eds. (Gif sur Yvette: Editions Frontieres)

Miller, J. S. 1981,Pub.A.S.P, 93, 681.

Neugebauer, G., Soifer, B. T., and Miley, G. K., 1982,Astrophys. J. Lett., 295,L27.

Neugebauer, G., Miley, G. K., Soifer, B. T. and Clegg, P. E., 1986,Astrophys. J., 308,815

Norman, C. and Scoville, N. Z., 1988, Astrophys. J., 332, 124.

Sanders, D. B., Soifer, B. T., Elias, J. H., Madore, B. F., Matthews, K., Neugebauer, G., and Scoville, N. Z., 1988, Astrophys. J., 325,74

Sekiguchi, K., 1987, Astrophys. J., 316,145

Soifer, B. T., Boehmer, L., Neugebauer, G., and Sanders, D. B., 1989,Astron. J., 98,766

Sopp, H. M. and Alexander, P., 1989, Astrophys. Space Sc., 157,287 

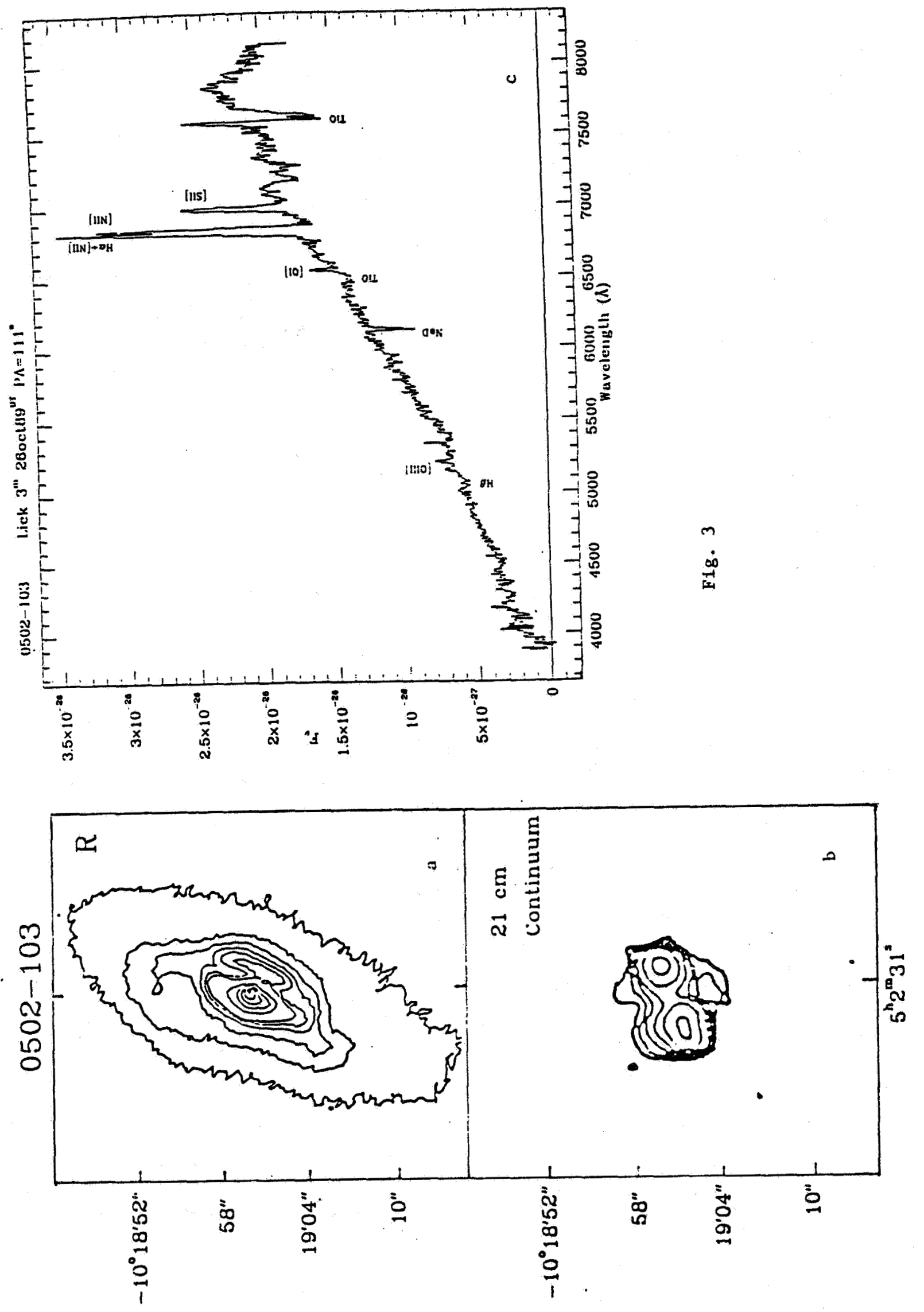


\section{DISCUSSION}

Norris: What is the distribution of far-IR color in your radioloud sample? It would be very interesting to see if your sample consists only of warm (Seyfert-like) objects, or whether cooler, starburst galaxies could also be radio-loud.

Dey: So far 6 out of 39 galaxies have HII-region type spectra. The colors of our sample scatter over a large range, but on average are warmer than any flux-limited IRAS sample. There are some objects whose IRAS colors are similar to those of starbursts. 